\title{
Evidence for Oscillation of Atmospheric Neutrinos
}

Y. Fukuda, ${ }^{1}$ T. Hayakawa, ${ }^{1}$ E. Ichihara,${ }^{1}$ K. Inoue,${ }^{1}$ K. Ishihara,${ }^{1}$ H. Ishino,,${ }^{1}$ Y. Itow, ${ }^{1}$ T. Kajita,,${ }^{1}$ J. Kameda ${ }^{1}$ S. Kasuga,${ }_{1}^{1}$ K. Kobayashi, ${ }^{1}$ Y. Kobayashi, ${ }^{1}$ Y. Koshio, ${ }^{1}$ M. Miura, ${ }^{1}$ M. Nakahata, ${ }^{1}$ S. Nakayama, ${ }^{1}$ A. Okada,${ }^{1}$ K. Okumura, ${ }_{1}^{1}$ N. Sakurai, ${ }^{1}$ M. Shiozawa, ${ }^{1}$ Y. Suzuki, ${ }^{1}$ Y. Takeuchi, ${ }^{1}$ Y. Totsuka, ${ }^{1}$ S. Yamada, ${ }^{1}$ M. Earl, ${ }^{2}$ A. Habig, ${ }^{2}$ E. Kearns, ${ }^{2}$ M. D. Messier, ${ }^{2}$ K. Scholberg, ${ }^{2}$ J. L. Stone, ${ }^{2}$ L. R. Sulak, ${ }^{2}$ C. W. Walter, ${ }^{2}$ M. Goldhaber, ${ }^{3}$ T. Barszczxak, ${ }^{4}$ D. Casper, ${ }^{4}$ W. Gajewski, ${ }^{4}$ P. G. Halverson, ${ }^{4}, *$ J. Hsu, ${ }^{4}$ W. R. Kropp,${ }^{4}$ L. R. Price,${ }^{4}$ F. Reines,${ }^{4}$ M. Smy,${ }^{4}$ H. W. Sobel, ${ }^{4}$ M. R. Vagins, ${ }^{4}$ K. S. Ganezer, ${ }^{5}$ W.E. Keig, ${ }^{5}$ R. W. Ellsworth, ${ }^{6}$ S. Tasaka,${ }^{7}$ J. W. Flanagan, ${ }^{8,}{ }^{\dagger}$ A. Kibayashi, ${ }^{8}$ J. G. Learned,${ }^{8}$ S. Matsuno, ${ }^{8}$ V. J. Stenger, ${ }^{8}$ D. Takemori, ${ }^{8}$ T. Ishii, ${ }^{9}$ J. Kanzaki, ${ }^{9}$ T. Kobayashi, ${ }^{9}$ S. Mine, ${ }^{9}$ K. Nakamura, ${ }^{9}$ K. Nishikawa, ${ }^{9}$ Y. Oyama,${ }^{9}$ A. Sakai,${ }^{9}$ M. Sakuda,,${ }^{9}$ O. Sasaki, ${ }^{9}$ S. Echigo, ${ }^{10}$ M. Kohama,${ }^{10}$ A. T. Suzuki, ${ }^{10}$ T. J. Haines, ${ }^{11,4}$ E. Blaufuss, ${ }^{12}$ B. K. Kim, ${ }^{12}$ R. Sanford,${ }^{12}$ R. Svoboda, ${ }^{12}$ M. L. Chen, ${ }^{13}$ Z. Conner, ${ }^{13, *}$ J. A. Goodman, ${ }^{13}$ G. W. Sullivan, ${ }^{13}$ J. Hill, ${ }^{14}$ C. K. Jung, ${ }^{14}$ K. Martens, ${ }^{14}$ C. Mauger, ${ }^{14}$ C. McGrew,${ }^{14}$ E. Sharkey,${ }^{14}$ B. Viren, ${ }^{14}$ C. Yanagisawa,${ }^{14}$ W. Doki, ${ }^{15}$ K. Miyano,${ }^{15}$ H. Okazawa, ${ }^{15}$ C. Saji,${ }^{15}$ M. Takahata, ${ }^{15}$ Y. Nagashima, ${ }^{16}$ M. Takita, ${ }^{16}$ T. Yamaguchi, ${ }^{16}$ M. Yoshida, ${ }^{16}$ S. B. Kim,,${ }^{17}$ M. Etoh,${ }^{18}$ K. Fujita, ${ }^{18}$ A. Hasegawa, ${ }^{18}$ T. Hasegawa, ${ }^{18}$ S. Hatakeyama, ${ }^{18}$ T. Iwamoto, ${ }^{18}$ M. Koga,${ }^{18}$ T. Maruyama, ${ }^{18}$ H. Ogawa, ${ }^{18}$ J. Shirai, ${ }^{18}$ A. Suzuki, ${ }^{18}$ F. Tsushima, ${ }^{18}$ M. Koshiba, ${ }^{19}$ M. Nemoto,${ }^{20}$ K. Nishijima, ${ }^{20}$ T. Futagami, ${ }^{21}$ Y. Hayato, ${ }^{21,}{ }^{\S}$ Y. Kanaya, ${ }^{21}$ K. Kaneyuki, ${ }^{21}$ Y. Watanabe, ${ }^{21}$ D. Kielczewska, ${ }^{22,4}$ R. A. Doyle, ${ }^{23}$ J. S. George,${ }^{23}$ A. L. Stachyra, ${ }^{23}$ L. L. Wai, ${ }^{23, \|}$ R. J. Wilkes, ${ }^{23}$ and K. K. Young ${ }^{23}$ (Super-Kamiokande Collaboration)

${ }^{1}$ Institute for Cosmic Ray Research, University of Tokyo, Tanashi, Tokyo, 188-8502, Japan

${ }^{2}$ Department of Physics, Boston University, Boston, Massachusetts 02215

${ }^{3}$ Physics Department, Brookhaven National Laboratory, Upton, New York 11973

${ }^{4}$ Department of Physics and Astronomy, University of California at Irvine, Irvine, California 92697-4575

${ }^{5}$ Department of Physics, California State University, Dominguez, Hills, Carson, California 90747

${ }^{6}$ Department of Physics, George Mason University, Fairfax, Virginia 22030

${ }^{7}$ Department of Physics, Gifu University, Gifu, Gifu 501-1193, Japan

${ }^{8}$ Department of Physics and Astronomy, University of Hawaii, Honolulu, Hawaii 96822

${ }^{9}$ Institute of Particle and Nuclear Studies, High Energy Accelerator Research Organization (KEK), Tsukuba, Ibaraki 305-0801, Japan

${ }^{10}$ Department of Physics, Kobe University, Kobe, Hyogo 657-8501, Japan

${ }^{11}$ Physics Division, P-23, Los Alamos National Laboratory, Los Alamos, New Mexico 87544

${ }^{12}$ Department of Physics and Astronomy, Louisiana State University, Baton Rouge, Louisiana 70803

${ }^{13}$ Department of Physics, University of Maryland, College Park, Maryland 20742

${ }^{14}$ Department of Physics and Astronomy, State University of New York, Stony Brook, New York 11794-3800

${ }^{15}$ Department of Physics, Niigata University, Niigata, Niigata 950-2181, Japan

${ }^{16}$ Department of Physics, Osaka University, Toyonaka, Osaka 560-0043, Japan

${ }^{17}$ Department of Physics, Seoul National University, Seoul 151-742, Korea

${ }^{18}$ Department of Physics, Tohoku University, Sendai, Miyagi 980-8578, Japan

${ }^{19}$ The University of Tokyo, Tokyo 113-0033, Japan

${ }^{20}$ Department of Physics, Tokai University, Hiratsuka, Kanagawa 259-1292, Japan

${ }^{21}$ Department of Physics, Tokyo Institute of Technology, Meguro, Tokyo 152-8551, Japan

${ }^{22}$ Institute of Experimental Physics, Warsaw University, 00-681 Warsaw, Poland

${ }^{23}$ Department of Physics, University of Washington, Seattle, Washington 98195-1560 (Received 6 July 1998)

We present an analysis of atmospheric neutrino data from a 33.0 kton yr (535-day) exposure of the Super-Kamiokande detector. The data exhibit a zenith angle dependent deficit of muon neutrinos which is inconsistent with expectations based on calculations of the atmospheric neutrino flux. Experimental biases and uncertainties in the prediction of neutrino fluxes and cross sections are unable to explain our observation. The data are consistent, however, with two-flavor $\nu_{\mu} \leftrightarrow \nu_{\tau}$ oscillations with $\sin ^{2} 2 \theta>$ 0.82 and $5 \times 10^{-4}<\Delta m^{2}<6 \times 10^{-3} \mathrm{eV}^{2}$ at $90 \%$ confidence level. [S0031-9007(98)06975-0]

PACS numbers: 14.60.Pq, 96.40.Tv

Atmospheric neutrinos are produced as decay products in hadronic showers resulting from collisions of cosmic rays with nuclei in the upper atmosphere. Production of electron and muon neutrinos is dominated by the processes $\pi^{+} \rightarrow \mu^{+}+\nu_{\mu}$ followed by $\mu^{+} \rightarrow e^{+}+\bar{\nu}_{\mu}+$ $\nu_{e}$ (and their charge conjugates) giving an expected ratio 
$\left(\equiv \nu_{\mu} / \nu_{e}\right)$ of the flux of $\nu_{\mu}+\bar{\nu}_{\mu}$ to the flux of $\nu_{e}+\bar{\nu}_{e}$ of about 2. The $\nu_{\mu} / \nu_{e}$ ratio has been calculated in detail with an uncertainty of less than $5 \%$ over a broad range of energies from 0.1 to $10 \mathrm{GeV}[1,2]$.

The $\nu_{\mu} / \nu_{e}$ flux ratio is measured in deep underground experiments by observing final state leptons produced via charged-current interactions of neutrinos on nuclei, $\nu+$ $N \rightarrow l+X$. The flavor of the final state lepton is used to identify the flavor of the incoming neutrino.

The measurements are reported as $R \equiv(\mu / e)_{\mathrm{DATA}} /$ $(\mu / e)_{\mathrm{MC}}$, where $\mu$ and $e$ are the number of muonlike $(\mu$-like) and electronlike ( $e$-like) events observed in the detector for both data and Monte Carlo simulations. This ratio largely cancels experimental and theoretical uncertainties, especially the uncertainty in the absolute flux. $R=1$ is expected if the physics in the Monte Carlo simulation accurately models the data. Measurements of significantly small values of $R$ have been reported by the deep underground water Cherenkov detectors Kamiokande [3,4], IMB [5], and recently by Super-Kamiokande [6,7]. Although measurements of $R$ by early iron-calorimeter experiments Fréjus [8] and NUSEX [9] with smaller data samples were consistent with expectations, the Soudan-2 iron-calorimeter experiment has reported observation of a small value of $R$ [10].

Neutrino oscillations have been suggested to explain measurements of small values of $R$. For a two-neutrino oscillation hypothesis, the probability for a neutrino produced in flavor state $a$ to be observed in flavor state $b$ after traveling a distance $L$ through a vacuum is

$$
P_{a \rightarrow b}=\sin ^{2} 2 \theta \sin ^{2}\left(\frac{1.27 \Delta m^{2}\left(\mathrm{eV}^{2}\right) L(\mathrm{~km})}{E_{\nu}(\mathrm{GeV})}\right),
$$

where $E_{\nu}$ is the neutrino energy, $\theta$ is the mixing angle between the flavor eigenstates and the mass eigenstates, and $\Delta m^{2}$ is the mass squared difference of the neutrino mass eigenstates. For detectors near the surface of the Earth, the neutrino flight distance, and thus the oscillation probability, is a function of the zenith angle of the neutrino direction. Vertically downward-going neutrinos travel about $15 \mathrm{~km}$, while vertically upward-going neutrinos travel about $13000 \mathrm{~km}$ before interacting in the detector. The broad energy spectrum and this range of neutrino flight distances make measurements of atmospheric neutrinos sensitive to neutrino oscillations with $\Delta m^{2}$ down to $10^{-4} \mathrm{eV}^{2}$. The zenith angle dependence of $R$ measured by the Kamiokande experiment at high energies has been cited as evidence for neutrino oscillations [4].

We present our analysis of 33.0 kton yr (535 days) of atmospheric neutrino data from Super-Kamiokande. In addition to measurements of small values of $R$ both above and below $\sim 1 \mathrm{GeV}$, we observed a significant zenith angle dependent deficit of $\mu$-like events. While no combination of known uncertainties in the experimental measurement or predictions of atmospheric neutrino fluxes is able to explain our data, a two-neutrino oscillation model of $\nu_{\mu} \leftrightarrow \nu_{x}$, where $\nu_{x}$ may be $\nu_{\tau}$ or a new, noninteracting "sterile" neutrino, is consistent with the observed flavor ratios and zenith angle distributions over the entire energy region.

Super-Kamiokande is a 50 kton water Cherenkov detector instrumented with 11146 photomultiplier tubes (PMTs) facing an inner 22.5 kton fiducial volume of ultrapure water. Interaction kinematics are reconstructed using the time and charge of each PMT signal. The inner volume is surrounded by a $\sim 2 \mathrm{~m}$ thick outer detector instrumented with 1885 outward-facing PMTs. The outer detector is used to veto entering particles and to tag exiting tracks.

Super-Kamiokande has collected a total of 4353 fully contained (FC) events and 301 partially contained (PC) events in a 33.0 kton yr exposure. FC events deposit all of their Cherenkov light in the inner detector while PC events have exiting tracks which deposit some Cherenkov light in the outer detector. For this analysis, the neutrino interaction vertex was required to have been reconstructed within the 22.5 kton fiducial volume, defined to be $>2 \mathrm{~m}$ from the PMT wall.

FC events were separated into those with a single visible Cherenkov ring and those with multiple Cherenkov rings. For the analysis of FC events, only single-ring events were used. Single-ring events were identified as $c$-like or $\mu$-like based on a likelihood analysis of light detected around the Cherenkov cone. The FC events were separated into "sub-GEV" $\left(E_{\mathrm{vis}}<1330 \mathrm{MeV}\right)$ and "multi-GeV" $\left(E_{\mathrm{vis}}>\right.$ $1330 \mathrm{MeV}$ ) samples, where $E_{\text {vis }}$ is defined to be the energy of an electron that would produce the observed amount of Cherenkov light. $E_{\mathrm{vis}}=1330 \mathrm{MeV}$ corresponds to $p_{\mu} \sim 1400 \mathrm{MeV} / c$.

In a full-detector Monte Carlo simulation, $88 \%$ (96\%) of the sub-GeV $e$-like ( $\mu$-like) events were $\nu_{e}\left(\nu_{\mu}\right)$ chargedcurrent interactions and $84 \%$ (99\%) of the multi-GeV $e$-like ( $\mu$-like) events were $\nu_{e}\left(\nu_{\mu}\right)$ charged-current (CC) interactions. PC events were estimated to be $98 \% \nu_{\mu}$ charged-current interactions; hence, all PC events were classified as $\mu$-like, and no single-ring requirement was made. Table I summarizes the number of observed events for both data and Monte Carlo as well as the $R$ values for the sub-GeV and multi-GeV samples. Further details of the detector, data selection, and event reconstruction used in this analysis are given elsewhere [6,7].

We have measured significantly small values of $R$ in both the sub-GeV and multi-GeV samples. Several sources of systematic uncertainties in these measurements have been considered. Cosmic ray induced interactions in the rock surrounding the detector have been suggested as a source of $e$-like contamination from neutrons, which could produce small $R$ values [11], but these backgrounds have been shown to be insignificant for large water Cherenkov detectors [12]. In particular, Super-Kamiokande has $4.7 \mathrm{~m}$ of water surrounding the fiducial volume; this distance corresponds to roughly 5 hadronic interaction lengths and 13 radiation lengths. Distributions of event vertices 
TABLE I. Summary of the sub-GeV, multi-GeV, and PC event samples compared with the Monte Carlo prediction based on the neutrino flux calculation of Ref. [2].

\begin{tabular}{lcc}
\hline \hline & Data & Monte Carlo \\
\hline Sub-GeV & & \\
Single-ring & 2389 & 2622.6 \\
$e$-like & 1231 & 1049.1 \\
$\mu$-like & 1158 & 1573.6 \\
Multi-ring & 911 & 980.7 \\
Total & 3300 & 3603.3 \\
& $R=0.63 \pm 0.03$ (stat.) \pm 0.05 (syst.) \\
\hline Multi-Gev & & \\
Single-ring & 520 & 531.7 \\
$e$-like & 290 & 236.0 \\
$\mu$-like & 230 & 295.7 \\
Multi-ring & 533 & 560.1 \\
Total & 1053 & 1091.8 \\
\hline Partially contained & 301 & 371.6 \\
& $R_{\mathrm{FC}+\mathrm{PC}}=0.65 \pm 0.05$ (stat.) \pm 0.08 (syst.) \\
\hline \hline
\end{tabular}

exhibit no excess of $e$-like events close to the fiducial boundary [6,7].

The prediction of the ratio of the $\nu_{\mu}$ flux to the $\nu_{e}$ flux is dominated by the well-understood decay chain of mesons and contributes less than $5 \%$ of the uncertainty in $R$. Different neutrino flux models vary by about $\pm 20 \%$ in the prediction of absolute rates, but the ratio is robust [13]. Uncertainties in $R$ due to a difference in cross sections for $\nu_{e}$ and $\nu_{\mu}$ have been studied [14]; however, lepton universality prevents any significant difference in cross sections at energies much above the muon mass and thus errors in cross sections could not produce a small value of $R$ in the multi-GeV energy range. Particle identification was estimated to be $\gtrsim 98 \%$ efficient for both $\mu$-like and $e$-like events based on Monte Carlo studies. Particle identification was also tested in Super-Kamiokande on Michel electrons and stopping cosmic-ray muons and the $\mu$-like and $e$-like events used in this analysis are clearly separated [6]. The particle identification programs in use have also been tested using beams of electrons and muons incident on a water Cherenkov detector at KEK [15]. The data have been analyzed independently by two groups, making the possibility of significant biases in data selection or event reconstruction algorithms remote $[6,7]$. Other explanations for the small value of $R$, such as contributions from nucleon decays [16], can be discounted as they would not contribute to the zenith angle effects described below.

We estimate the probability that the observed $\mu / e$ ratios could be due to statistical fluctuation is less than $0.001 \%$ for sub-GeV $R$ and less than $1 \%$ for multi-GeV $R$.

The $\mu$-like data exhibit a strong asymmetry in zenith angle $(\Theta)$ while no significant asymmetry is observed in the $e$-like data [7]. The asymmetry is defined as $A=$

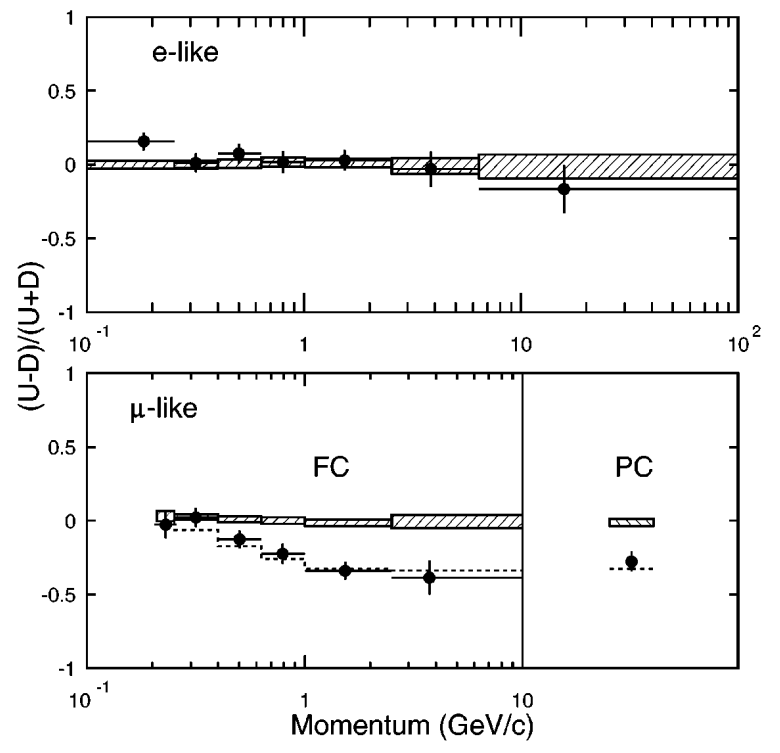

FIG. 1. The $(U-D) /(U+D)$ asymmetry as a function of momentum for $\mathrm{FC} e$-like and $\mu$-like events and $\mathrm{PC}$ events. While it is not possible to assign a momentum to a PC event, the PC sample is estimated to have a mean neutrino energy of $15 \mathrm{GeV}$. The Monte Carlo expectation without neutrino oscillations is shown in the hatched region with statistical and systematic errors added in quadrature. The dashed line for $\mu$-like is the expectation for $\nu_{\mu} \leftrightarrow \nu_{\tau} \quad$ oscillations with $\left(\sin ^{2} 2 \theta=1.0, \Delta m^{2}=2.2 \times\right.$ $\left.10^{-3} \mathrm{eV}^{2}\right)$.

$(U-D) /(U+D)$ where $U$ is the number of upwardgoing events $(-1<\cos \Theta<-0.2)$ and $D$ is the number of downward-going events $(0.2<\cos \Theta<1)$. The asymmetry is expected to be near zero independent of the flux model for $E_{\nu}>1 \mathrm{GeV}$, above which effects due to the Earth's magnetic field on cosmic rays are small. Based on a comparison of results from our full Monte Carlo simulation using different flux models $[1,2]$ as inputs, treatment of geomagnetic effects results in an uncertainty of roughly \pm 0.02 in the expected asymmetry of $e$-like and $\mu$-like sub-GeV events and less than \pm 0.01 for multi-GeV events. Studies of decay electrons from stopping muons show at most a $\pm 0.6 \%$ up-down difference in Cherenkov light detection [17].

Figure 1 shows $A$ as a function of momentum for both $e$-like and $\mu$-like events. In the present data, the asymmetric as a function of momentum for $e$-like events is consistent with expectations, while the $\mu$-like asymmetry at low momentum is consistent with zero but significantly deviates form expectations at higher momentum. The average angle between the final state lepton direction and the incoming neutrino direction is $55^{\circ}$ at $p=400 \mathrm{MeV} / c$ and $20^{\circ}$ at $1.5 \mathrm{GeV} / c$. At the lower momenta in Fig. 1, the possible asymmetry of the neutrino flux is largely washed out. We have found no detector bias differentiating $e$-like and $\mu$-like events that could explain an asymmetry in $\mu$-like events but not in $e$-like events [7]. 
Considering multi-GeV (FC $+\mathrm{PC})$ muons alone, the measured asymmetry, $A=-0.296 \pm 0.048 \pm 0.01 \mathrm{de}-$ viates from zero by more than 6 standard deviations.

We have examined the hypotheses of two-flavor $\nu_{\mu} \leftrightarrow$ $\nu_{e}$ and $\nu_{\mu} \leftrightarrow \nu_{\tau}$ oscillation models using a $\chi^{2}$ comparison of data and Monte Carlo, allowing all important Monte Carlo parameters to vary weighted by their expected uncertainties.

The data were binned by particle type, momentum, and $\cos \Theta$. A $\chi^{2}$ is defined as

$$
\chi^{2}=\sum_{\cos \Theta, p}\left(N_{\mathrm{DATA}}-N_{\mathrm{MC}}\right)^{2} / \sigma^{2}+\sum_{j} \epsilon_{j}^{2} / \sigma_{j}^{2},
$$

where the sum is over five bins equally spaced in $\cos \Theta$ and seven momentum bins for both $e$-like events and $\mu$-like plus PC events (70 bins total). The statistical error, $\sigma$, accounts for both data statistics and the weighted Monte Carlo statistics. $N_{\text {DATA }}$ is the measured number of events in each bin. $N_{\mathrm{MC}}$ is the weighted sum of Monte Carlo events:

$$
N_{\mathrm{MC}}=\frac{\mathcal{L}_{\mathrm{DATA}}}{\mathcal{L}_{\mathrm{MC}}} \sum_{\mathrm{MC} \text { events }} w .
$$

$\mathcal{L}_{\mathrm{DATA}}$ and $\mathcal{L}_{\mathrm{MC}}$ are the data and Monte Carlo live times. For each Monte Carlo event, the weight $w$ is given by

$$
\begin{aligned}
w= & (1+\alpha)\left(E_{\nu}^{i} / E_{0}\right)^{\delta}\left(1+\eta_{s, m} \cos \Theta\right) \\
& \times f_{e, \mu}\left(\sin ^{2} 2 \theta, \Delta m^{2},(1+\lambda) L / E_{\nu}\right) \\
& \times \begin{cases}\left(1-\beta_{s} / 2\right) & \text { sub-GeV } e \text {-like, } \\
\left(1+\beta_{s} / 2\right) & \text { sub-GeV } \mu \text {-like, } \\
\left(1-\beta_{m} / 2\right) & \text { multi-GeV } e \text {-like, } \\
\left(1+\beta_{m} / 2\right)\left(1-\frac{\rho}{2} \frac{N_{P C}}{N_{\mu}}\right) & \text { multi-GeV } \mu \text {-like, } \\
\left(1+\beta_{m} / 2\right)\left(1+\frac{\rho}{2}\right) & \text { PC } .\end{cases}
\end{aligned}
$$

$E_{\nu}^{i}$ is the average neutrino energy in the $i$ th momentum bin; $E_{0}$ is an arbitrary reference energy (taken to be $2 \mathrm{GeV}$ ); $\eta_{s}\left(\eta_{m}\right)$ is the up-down uncertainty of the event rate in the sub-GeV (multi-GeV) energy range; $N_{\mathrm{PC}}$ is the total number of Monte Carlo PC events; and $N_{\mu}$ is the total number of Monte Carlo FC multi-GeV muons. The factor $f_{e, \mu}$ weights an event accounting for the initial neutrino fluxes (in the case of $\nu_{\mu} \leftrightarrow \nu_{e}$ ), oscillation parameters, and $L / E_{\nu}$. The meaning of the Monte Carlo fit parameters, $\alpha$ and $\epsilon_{j} \equiv\left(\beta_{s}, \beta_{m}, \delta, \rho, \lambda, \eta_{s}, \eta_{m}\right)$ and their assigned uncertainties, $\sigma_{j}$, are summarized in Table II. The overall normalization, $\alpha$, was allowed to vary freely. The uncertainty in the Monte Carlo $L / E_{\nu}$ ratio $(\lambda)$ was conservatively estimated based on the uncertainty in an absolute energy scale, uncertainty in neutrino-lepton angular and energy correlations, and the uncertainty in production height. The oscillation simulations used profiles of neutrino production heights calculated in Ref. [18], which account for the competing factors of production, propagation, and decay of muons and mesons through the atmosphere.
TABLE II. Summary of Monte Carlo fit parameters. Bestfit values for $\nu_{\mu} \leftrightarrow \nu_{\tau}\left(\Delta m^{2}=2.2 \times 10^{-3} \mathrm{eV}^{2}, \sin ^{2} 2 \theta=\right.$ 1.0) and estimated uncertainties are given. (*) The overall normalization $(\alpha)$ was estimated to have a $25 \%$ uncertainty but was fitted as a free parameter.

\begin{tabular}{llcc}
\hline \hline & Monte Carlo fit parameters & Best fit & Uncertainty \\
\hline$\alpha$ & Overall normalization & $15.8 \%$ & $(*)$ \\
$\delta$ & $E_{\nu}$ spectral index & 0.006 & $\sigma_{\delta}=0.05$ \\
$\beta_{s}$ & Sub-GeV $\mu / e$ ratio & $-6.3 \%$ & $\sigma_{s}=8 \%$ \\
$\beta_{m}$ & Multi-GeV $\mu / e$ ratio & $-11.8 \%$ & $\sigma_{m}=12 \%$ \\
$\rho$ & Relative norm. of PC to FC & $-1.8 \%$ & $\sigma_{p}=8 \%$ \\
$\lambda$ & $L / E_{\nu}$ & $3.1 \%$ & $\sigma_{\lambda}=15 \%$ \\
$\eta_{s}$ & Sub-GeV up-down & $2.4 \%$ & $\sigma_{\eta}^{s}=2.4 \%$ \\
$\eta_{m}$ & Multi-GeV up-down & $-0.09 \%$ & $\sigma_{\eta}^{m}=2.7 \%$ \\
\hline \hline
\end{tabular}

For $\nu_{\mu} \leftrightarrow \nu_{e}$, effects of matter on neutrino propagation through the Earth were included following Ref. [19,20]. Because of the small number of events expected from $\tau$ production, the effects of $\tau$ appearance and decay were neglected in simulations of $\nu_{\mu} \leftrightarrow \nu_{\tau}$. A global scan was made on a $\left(\sin ^{2} 2 \theta, \log \Delta m^{2}\right)$ grid minimizing $\chi^{2}$ with respect to $\alpha, \beta_{s}, \beta_{m}, \delta, \rho, \lambda, \eta_{s}$, and $\eta_{m}$ at each point.

The best fit to $\nu_{\mu} \leftrightarrow \nu_{\tau}$ oscillations, $\chi_{\min }^{2}=65.2 /$ $67 \mathrm{DOF}$, was obtained at $\left(\sin ^{2} 2 \theta=1.0, \Delta m^{2}=2.2 \times\right.$ $\left.10^{-3} \mathrm{eV}^{2}\right)$ inside the physical region $\left(0 \leq \sin ^{2} 2 \theta \leq 1\right)$. The best-fit values of the Monte Carlo parameters (summarized in Table II) were all within their expected errors. The global minimum occurred slightly outside of the physical region at $\left(\sin ^{2} 2 \theta=1.05, \Delta m^{2}=2.2 \times 10^{-3} \mathrm{eV}^{2}\right.$, $\chi_{\min }^{2}=64.8 / 67$ DOF). The contours of the $68 \%, 90 \%$, and $99 \%$ confidence intervals are located at $\chi_{\min }^{2}+2.6$, 5.0 , and 9.6 based on the minimum inside the physical region [21]. Thee contours are shown in Fig. 2. The region near $\chi^{2}$ minimum is rather flat and has many local minima so that inside the $68 \%$ interval the best-fit $\Delta m^{2}$ is not well-constrained. Outside of the $99 \%$ allowed region the $\chi^{2}$ increases rapidly. We obtained $\chi^{2}=135 / 69$ DOF, when calculated at $\sin ^{2} 2 \theta=0, \Delta m^{2}=0$ (i.e., assuming no oscillations).

For the test of $\nu_{\mu} \leftrightarrow \nu_{e}$ oscillations, we obtained a relatively poor fit; $\chi_{\min }^{2}=87.8 / 67 \mathrm{DOF}$, at $\left(\sin ^{2} 2 \theta=\right.$ $0.93, \Delta m^{2}=3.2 \times 10^{-3} \mathrm{eV}^{2}$ ). The expected asymmetry of the multi-GeV $e$-like events for the best-fit $\nu_{\mu} \leftrightarrow$ $\nu_{e}$ oscillation hypothesis, $A=0.205$, differs from the measured asymmetry, $A=-0.036 \pm 0.067 \pm 0.02$, by 3.4 standard deviations. We conclude that the $\nu_{\mu} \leftrightarrow \nu_{e}$ hypothesis is not favored.

The zenith angle distributions for the FC and PC samples are shown in Fig. 3. The data are compared to the Monte Carlo expectation (no oscillations, hatched region) and the best-fit expectation for $\nu_{\mu} \leftrightarrow \nu_{\tau}$ oscillations (bold line).

We also estimated the oscillation parameters considering the $R$ measurement and the zenith angle shape separately. The $90 \%$ confidence level allowed regions for each 


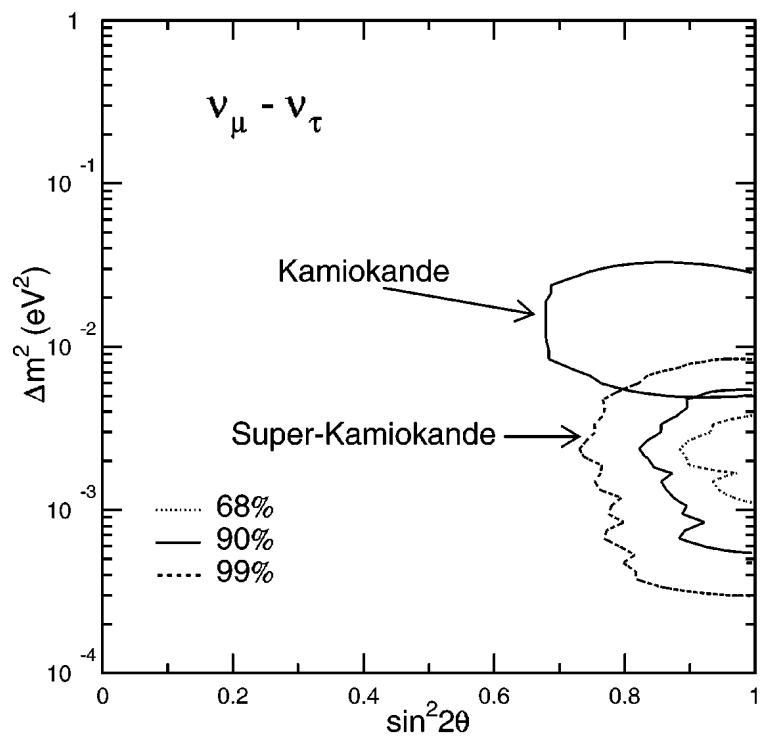

FIG. 2. The $68 \%, 90 \%$, and $99 \%$ confidence intervals are shown for $\sin ^{2} 2 \theta$ and $\Delta m^{2}$ for $\nu_{\mu} \leftrightarrow \nu_{\tau}$ two-neutrino oscillations based on 33.0 kton yr of Super-Kamiokande data. The $90 \%$ confidence interval obtained by the Kamiokande experiment is also shown.

case overlapped at $1 \times 10^{-3}<\Delta m^{2}<4 \times 10^{-3} \mathrm{eV}^{2}$ for $\sin ^{2} 2 \theta=1$.

As a cross-check of the above analyses, we have reconstructed the best estimate of the ratio $L / E_{\nu}$ for each event. The neutrino energy is estimated by applying a correction to the final state lepton momentum. Typi- cally, final state leptons with $p \sim 100 \mathrm{MeV} / c$ carry $65 \%$ of the incoming neutrino energy increasing to $\sim 85 \%$ at $p=1 \mathrm{GeV} / c$. The neutrino flight distance $L$ is estimated following Ref. [18] using the estimated neutrino energy and the reconstructed lepton direction and flavor. Figure 4 shows the ratio of FC data to Monte Carlo for $e$-like and $\mu$-like events with $p>400 \mathrm{MeV}$ as a function of $L / E_{\nu}$, compared to the expectation for $\nu_{\mu} \leftrightarrow \nu_{\tau}$ oscillations with our best-fit parameters. The $e$-like data show no significant variation in $L / E_{\nu}$, while the $\mu$-like events show a significant deficit at large $L / E_{\nu}$. At large $L / E_{\nu}$, the $\nu_{\mu}$ have presumably undergone numerous oscillations and have averaged out to roughly half the initial rate.

The asymmetry $A$ of the $e$-like events in the present data is consistent with expectations without neutrino oscillations and two-flavor $\nu_{e} \leftrightarrow \nu_{\mu}$ oscillations are not favored. This is in agreement with recent results from the $\mathrm{CHOOZ}$ experiment [22]. The LSND experiment has reported the appearance of $\nu_{e}$ in a beam of $\nu_{\mu}$ produced by stopped pions [23]. The LSND results do not contradict the present results if they are observing small mixing angles. With the best-fit parameters for $\nu_{\mu} \leftrightarrow \nu_{\tau}$ oscillations, we expect a total of only 15-20 events from $\nu_{\tau}$ chargedcurrent interactions in the data sample. Using the current sample, oscillations between $\nu_{\mu}$ and $\nu_{\tau}$ are indistinguishable from oscillations between $\nu_{\mu}$ and a noninteracting sterile neutrino.

Figure 2 shows the Super-Kamiokande results overlaid with the allowed region obtained by the Kamiokande
sub-GeV

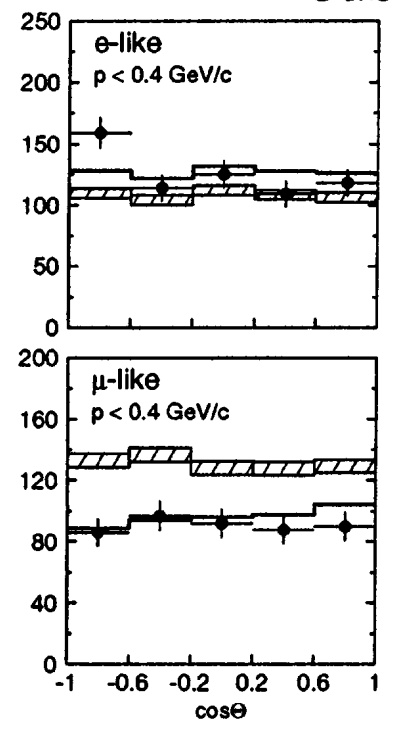

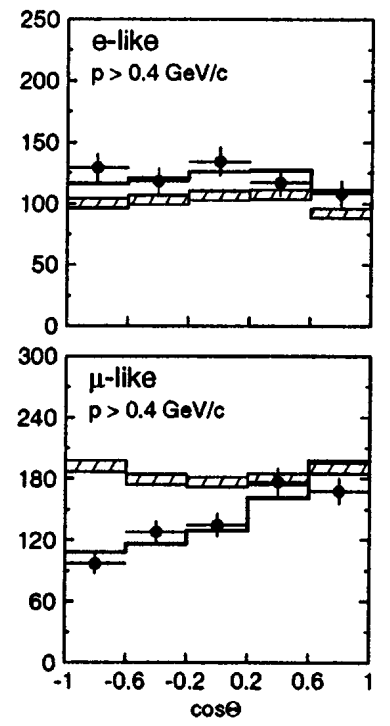

multi-GeV

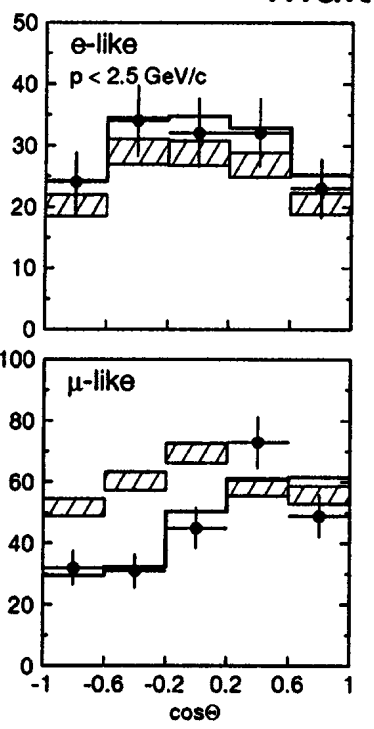

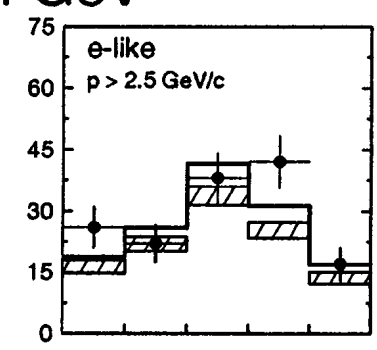

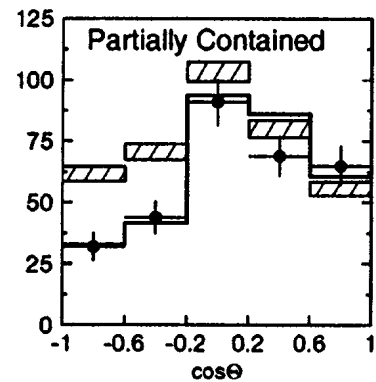

FIG. 3. Zenith angle distributions of $\mu$-like and $e$-like events for sub-GeV and multi-GeV data sets. Upward-going particles have $\cos \Theta<0$ and downward-going particles have $\cos \Theta>0$. Sub-GeV data are shown separately for $p<400 \mathrm{MeV} / c$ and $p>400 \mathrm{MeV} / c$. Multi-GeV $e$-like distributions are shown for $p<2.5$ and $p>2.5 \mathrm{GeV} / c$ and the multi-GeV $\mu$-like are shown separately for FC and PC events. The hatched region shows the Monte Carlo expectation for no oscillations normalized to the data live time with statistical errors. The bold line is the best-fit expectation for $\nu_{\mu} \leftrightarrow \nu_{\tau}$ oscillations with the overall flux normalization fitted as a free parameter. 


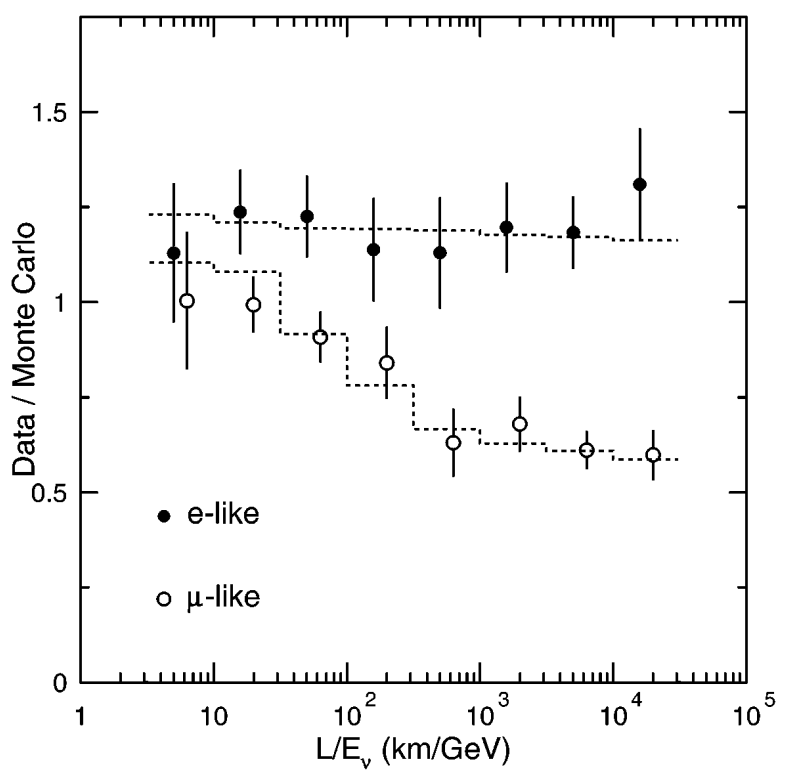

FIG. 4. The ratio of the number of FC data events to FC Monte Carlo events versus reconstructed $L / E_{\nu}$. The points show the ratio of observed data to $\mathrm{MC}$ expectation in the absence of oscillations. The dashed lines show the expected shape for $\nu_{\mu} \leftrightarrow \nu_{\tau}$ at $\Delta m^{2}=2.2 \times 10^{-3} \mathrm{eV}^{2}$ and $\sin ^{2} 2 \theta=$ 1. The slight $L / E_{\nu}$ dependence for $e$-like events is due to contamination $(2-7 \%)$ of $\nu_{\mu} \mathrm{CC}$ interactions.

experiment [4]. The Super-Kamiokande region favors lower values of $\Delta m^{2}$ than allowed by the Kamiokande experiment; however the $90 \%$ contours from both experiments have a region of overlap. Preliminary studies of upward-going stopping and through-going muons in Super-Kamiokande [24] give allowed regions consistent with the FC and PC event analysis reported in this paper.

Both the zenith angle distribution of $\mu$-like events and the value of $R$ observed in this experiment significantly differ from the best predictions in the absence of neutrino oscillations. While uncertainties in the flux prediction, cross sections, and experimental biases are ruled out as explanations of the observations, the present data are in good agreement with two-flavor $\nu_{\mu} \leftrightarrow \nu_{\tau}$ oscillations with $\sin ^{2} 2 \theta>0.82$ and $5 \times 10^{-4}<\Delta m^{2}<$ $6 \times 10^{-3} \mathrm{eV}^{2}$ at a $90 \%$ confidence level. We conclude that the present data give evidence for neutrino oscillations.

We gratefully acknowledge the cooperation of the Kamioka Mining and Smelting Company. The SuperKamiokande experiment was built and has been operated with funding from the Japanese Ministry of Education, Science, Sports and Culture, and the United States Department of Energy.
*Present address: NASA, JPL, Pasadena, CA 91109.

${ }^{\dagger}$ Present address: High Energy Accelerator Research Organization (KEK), Accelerator Laboratory, Tsukuba, Ibaraki 305-0801, Japan.

*Present address: University of Chicago, Enrico Fermi Institute, Chicago, IL 60637.

\$Present address: Institute of Particle and Nuclear Studies, High Energy Accelerator Research Organization (KEK), Tsukuba, Ibaraki 305-0801, Japan.

"Present address: Stanford University, Department of Physics, Stanford, CA 94305.

[1] G. Barr et al., Phys. Rev. D 39, 3532 (1998); V. Agrawal et al., Phys. Ref. D 53, 1314 (1996); T. K. Gaisser and T. Stanev, in Proceedings of the International Cosmic Ray Conference, Rome, Italy, 1995 (Arti Grafiche, Urbino, 1995), Vol. 1, p. 694.

[2] M. Honda et al., Phys. Lett. B 248, 193 (1990); M. Honda et al., Phys. Rev. D 52, 4985 (1995).

[3] K. S. Hirata et al., Phys. Lett. B 205, 416 (1988); K. S. Hirata et al., Phys. Lett. B 280, 146 (1992).

[4] Y. Fukuda et al., Phys. Lett. B 335, 237 (1994).

[5] D. Casper et al., Phys. Rev. Lett. 66, 2561 (1991); R. Becker-Szendy et al., Phys. Rev. D 46, 3720 (1992).

[6] Super-Kamiokande Collaboration, Y. Fukuda et al., hepex/9803006.

[7] Super-Kamiokande Collaboration, Y. Fukuda, et al., hepex/9805006.

[8] K. Daum et al., Z. Phys. C 66, 417 (1995).

[9] M. Aglietta et al., Europhys. Lett. 8, 611 (1989).

[10] W. W. M. Allison et al., Phys. Lett. B 391, 491 (1997); T. Kafka, inProceedings of the 5th International Workshop on Topics in Astroparticle and Underground Physics, Gran Sasso, Italy, 1997 (to be published).

[11] O. G. Ryazhskaya, JETP Lett. 60, 617 (1994); JETP Lett. 61, 237 (1995).

[12] Y. Fukuda et al., Phys. Lett. B 388, 397 (1996).

[13] T. K. Gaisser et al., Phys. Rev. D 54, 5578 (1996).

[14] J. Engel et al., Phys. Rev. D 48, 3048 (1993).

[15] S. Kasuga et al., Phys. Lett. B 374, 238 (1996).

[16] W. A. Mann, T. Kafka, and W. Leeson, Phys. Lett. B 291, 200 (1992).

[17] This represents an improvement from Refs. [6,7] due to improved calibration.

[18] T. K. Gaisser and T. Stanev, Phys. Rev. D 57, 1977 (1998).

[19] L. Wolfenstein, Phys. Rev. D 17, 2369 (1978).

[20] S. P. Mikheyev and A. Y. Smirnov, Sov. J. Nucl. Phys. 42, 1441 (1985); S. P. Mikheyev and A. Y. Smirnov, Nuovo Cimento Soc. Ital. Fis. 9C, 17 (1986); S. P. Mikheyev and A. Y. Smirnov, Sov. Phys.-Usp. 30, 759 (1987).

[21] Based on a two-dimensional extension of the method from the Particle Data Group, R. M. Barnett et al., Phys. Rev. D 54, 1 (1996); see sec. 28.6, p. 162.

[22] M. Apollonio et al., Phys. Lett. B 420, 397 (1998).

[23] C. Athanassopoulos et al., Phys. Rev. C 54, 2685 (1996); Phys. Rev. Lett. 77, 3082 (1996).

[24] T. Kajita, in Proceedings of the XVIIIth International Conference on Neutrino Physics and Astrophysics, Takayama, Japan, 1998 (to be published). 